\title{
An Efficient Algorithm for Electrically Large Reflectarray Antenna Design Automation
}

\author{
Ghulam Ahmad, Tim W. C. Brown, Craig I. \\ Underwood \\ Faculty of Engineering and Physical Sciences (FEPS) \\ University of Surrey \\ Guildford, United Kingdom \\ \{g.ahmad, t.brown, c.underwood\}@surrey.ac.uk
}

\author{
Tian Hong Loh \\ Time, Quantum and Electromagnetics Division \\ National Physical Laboratory (NPL) \\ Teddington, United Kingdom \\ tian.loh@npl.co.uk
}

\begin{abstract}
Reflectarrays are becoming a potentially attractive replacement of parabolic reflectors for high gain requirements. A large reflectarray consists of thousands of elements. To predict their performance a simulation model is required which is very cumbersome to build manually due to a large number of elements. It takes exhaustive efforts, keen attention to details and significant amount of time to build such a simulation model. When several iterations of modelling are required it worsens the issue even further. We have presented here an algorithm as an automated solution to this problem by interfacing Matlab ${ }^{\circledR}$ with an electromagnetic simulation software. It is very generic, time efficient and makes the modelling easy with least intervention of the designer.
\end{abstract}

Keywords-Unit Cells, Reflectarray, Visual Basic, CST, Matlab

\section{INTRODUCTION}

A need for millimeter wave (mm-wave) high gain smart antennas to achieve wide angle beam steering at lower system losses make reflectarrays a potential candidate solution for next generation communication systems. A reflectarray adapts the feeding mechanism of parabolic reflectors and maintains the planar profile of phased arrays to accomplish high gain and wide angle beam steering at lower losses. To accurately predict the performance of a reflectarray it is required to simulate it in a suitable electromagnetic (EM) simulation software e.g. CST Microwave Studio, HFSS, or FEKO to name a few. Such simulations require building the reflectarray structure in the chosen simulation package. An electrically large reflectarray consists of thousands of elements known as unit cells. The design parameters of a unit cell are selected based on reflection phase curves e.g. length and width of patch in a variable length patch approach. These unit cells are arranged in a particular pattern to generate the collimated far field radiated beam in a certain direction. To prepare a simulation model for a large reflectarray is very cumbersome and it takes significant amount of time, and can be prone to human errors in forming the geometry. There is a need to automate this activity to reduce the laborious effort for antenna designers. To date there is no algorithm available at least in public domain to address this particular issue.

We present here a time efficient automated modelling of reflectarray structure by interfacing Matlab $^{\circledR}$ and CST

This research was jointly sponsored by Surrey Space Center; University of Surrey and National Physical Laboratory under the project code SSC_NPL_KB4545 as a part of research on mm-wave reflectarray antennas. The work of T. H. Loh was supported by the 2013 - 2016 and $2016-2017$ Quantum, Electromagnetics and Time program of the National Measurement Office, an Executive Agency of the U.K. Department for Business, Energy and Industrial Strategy, under Projects 116774 and 119575.
Microwave Studio. The algorithm is very generic and can also be adopted to build complex shapes other than the reflectarray. Depending on the user's choice the algorithm can be implemented in other programming softwares and simulation packages. We performed the required reflected phase angle calculations in Matlab $^{\circledR}$ to direct the radiated beam in a certain direction. CST was used to simulate the phase curves of unit cell with variable length approach. Once the required set of dimensions is decided, they are programmed in Matlab ${ }^{\circledR}$ to generate the structure of the reflectarray. We designed several passive arrays using this modelling technique and found it to be approximately 35 times more efficient than manual modelling. This technique is particularly useful where several design iterations are required.

Section II presents the algorithm flowchart to interface the programming software e.g. Matlab ${ }^{\circledR}$ with a EM simulation software e.g. CST using visual basic (VB) [1] scripts. Finally, section III concludes the contribution.

\section{Reflectarray Structure Modelling Algorithm}

In a reflectarray a constant phase of the entire reflected field is achieved in a plane normal to the direction of the desired pencil beam as expressed by [4]:

$$
\frac{2 \pi}{\lambda_{0}}\left(r_{m n}-\vec{R}_{m n} \cdot \hat{r}\right)-\Delta \Phi_{m n}=2 \pi N
$$

where $\lambda_{0}$ is free space wavelength, $\vec{r}_{m n}$ is the position vector of $m n^{\text {th }}$ element/unit cell relative to $(0,0, F), F$ is the focal length, $\vec{R}_{m n}$ is the position vector of $m n^{\text {th }}$ element relative to $(0,0,0)$, $\hat{r}_{b}$ is the direction vector of the desired pencil beam, $N=$ $1,2,3 \ldots$, and $\Delta \Phi_{m n}$ is the phase shift introduced by $m n^{\text {th }}$ unit cell of reflect array to its reflected field relative to the incident field.

Once the continuous phases for unit cell central locations in the reflectarray are calculated using (1), it is logical to discretize them into a set of phases that can be implemented in the reflectarray. The reflectarrays can benefit from implementing a discrete set of reflection phases and normally a three bit phase quantization results in a practically negligible loss in gain. When the set of discrete phases is selected, the required reflecting patch parameters/dimensions in a unit cell 
to produce it are found. For this purpose a unit cell is simulated in a EM simulation software to generate a set of phase curves by parametrizing the variables of unit cell e.g. patch length in a printed microstrip variable length based unit cell. From these simulation curves generated by EM software or by some other means a set of dimensions is selected corresponding to each phase in the set of required discretized phases. When the set of dimensions is known, a reflectarray can be constructed in EM simulation software for analyzing its RF performance. The construction of reflectarray from the known set of unit cell dimensions is a laborious process. We present below an automation procedure for this process using Matlab ${ }^{\circledR}$ and CST.

Matlab $^{\circledR}$ is very common among engineers and similarly it is true for CST Microwave Studio for RF and Microwave designers. Both provide an interface for VB scripts [2, 3]. Their interfacing is the solution to design automation. Fig. 1 displays the basic steps involved in the generation of a reflectarray structure. A Matlab ${ }^{\circledR}$ program calculates the required phases and the user selects the range of phase values which should correspond to a certain discrete phase. The unit cell parameters required to generate that particular discrete phase are fed to an EM simulator instance (CST project) via VB script in Matlab ${ }^{\circledR}$ for building a unit cell. This procedure is repeated for each location of unit cell in the reflectarray.

We have implemented the algorithm for various phase quantization bits using variable size square patches realized using generation of bricks in CST. The interface is syntax sensitive therefore needs a careful implementation. A designer can add as many phase discretization steps as deemed necessary without changing the construction time significantly. This is due to the fact that simple Matlab ${ }^{\circledR}$ commands run much faster as compared to its interface with CST. Our script stores editable history of objects which is a very desirable feature. Similarly, it arranges the unit cells in rows and columns as in visible geometry for easy access and modification at a later stage in a large set of unit cells. The implementation can be easily applied to circular or other shaped arrays by not calling the interface procedure for unit cells that are out of range. For other shapes user can use History List in CST for a particular object to understand the script required to build shapes of interest.

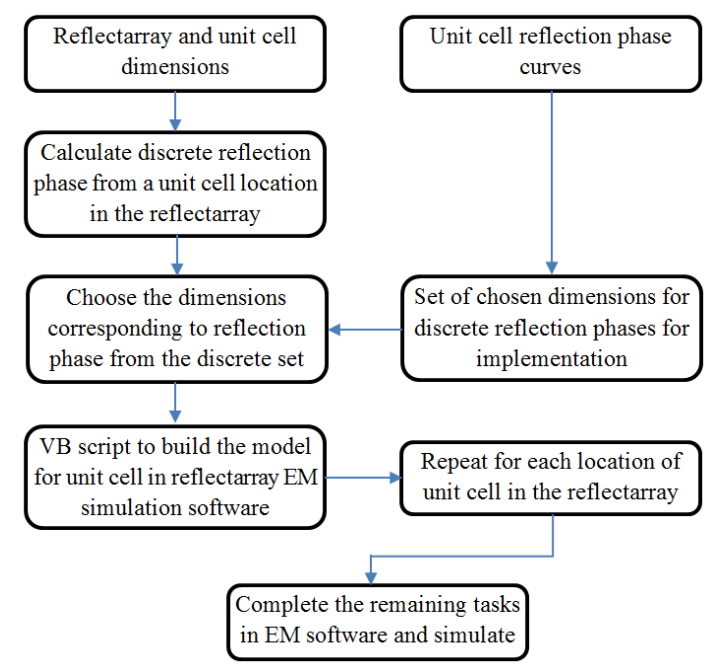

Fig. 1. Reflectarray structure model algorithm

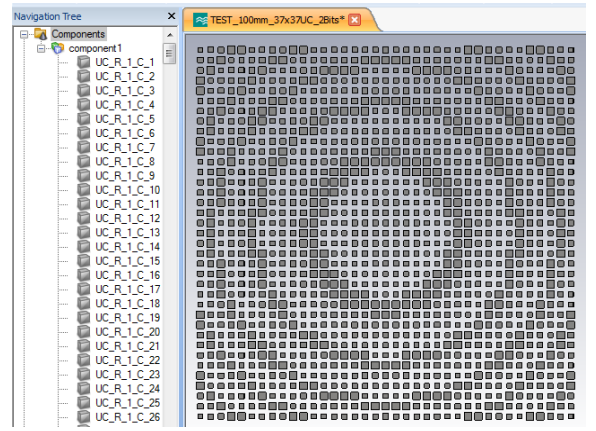

Fig. 2. A sample output from reflectarray structure model algorithm

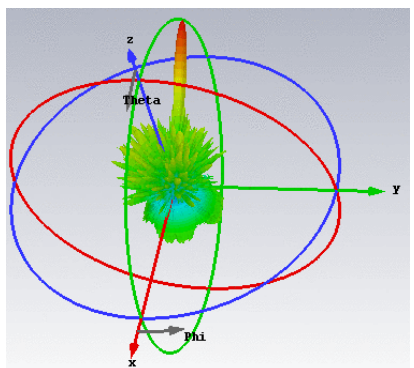

Fig. 3. A sample radiated far field at theta $55^{\circ}$ and phi $180^{\circ}$

A sample output from the script is shown in Fig. 2 for the structure of reflectarray. The grey patches are the unit cell patches to produce the required reflection phases from the particular locations. Once a geometry in CST has been generated using script, the designer needs only to select other simple parameters such as frequency, units and material specifications while also the feed horn model needs to be formed too. These steps can also be automated however left here for brevity. A sample simulated far field from the reflectarray prepared by our implementation of algorithm is shown in Fig. 3. It takes about a minute to manually build and set the required parameter values for each unit cell. For comparison our model took around 40 minutes on a normal desktop computer to build a reflectarray containing $37 \times 37$ unit cells which is drastically less as compared to the time taken if done manually.

\section{CONCLUSION}

An efficient automated algorithm for building a software EM simulation model of electrically large reflectarrays has been revealed in this contribution. For elaboration we used CST and Matlab ${ }^{\circledR}$ which were interfaced using visual basic scripts. The algorithm can be adapted to other EM simulators which support such scripting interfaces e.g. HFSS. By having such a small piece of script enables building very large structures automatically which would have taken days to accomplish otherwise.

\section{REFERENCES}

[1] (October, 2016). Visual Basic Tutorial. Available: http://www.vbtutor.net/vbtutor.html

[2] M. Balk. (2007), CST STUDIO SUITETM Introduction in VBA Macro usage and programming. Available: https://www.cst.com/Content/Events/UGM2007/02-Balk.pdf

[3] Y.-D. Wang and L. Bo, "Integrations between Visual Basic and MATLAB," in 2013 5th International Conference on Computational Intelligence and Communication Networks, 2013.

[4] S. V. Hum and J. Perruisseau-Carrier, "Reconfigurable reflectarrays and array lenses for dynamic antenna beam control: A review," Antennas and Propagation, IEEE Transactions on, vol. 62, pp. 183-198, 2014. 